\title{
IEEE 802.11ac MU-MIMO Wireless LAN cells with Legacy Clients
}

\author{
Mamun Abu-Tair*, Saleem N. Bhatti** \\ * School of Computing and Information Engineering, Ulster University, UK \\ ${ }^{* *}$ School of Computer Science, University of St Andrews, UK \\ *m.abu-tair@ulster.ac.uk, ** saleem@st-andrews.ac.uk
}

\begin{abstract}
We provide an empirical evaluation of an IEEE 802.11ac Wireless Local Area Network (WLAN) cell with Multiple User Multiple Input Multiple Output (MU-MIMO) technology. We conducted our experiments on a testbed comprising consumer equipment under different office scenarios using $40 \mathrm{MHz}$ and $80 \mathrm{MHz}$ channels. This is the first performance study of MU-MIMO with 802.11ac in an operational scenario using a commercial access point. We find that, for clients that do not support MU-MIMO, operating in a cell that has MU-MIMO enabled may result in reduced performance.
\end{abstract}

\section{INTRODUCTION}

Increasing network capacity is considered one of the important objectives of the last decade. Nowadays, Gigabit Ethernet cards and switches are found in personal computer and network infrastructures. For wireless networks, the restriction of shared media access is one of the major problems of increasing network capacity. With arrival of Multiple Input Multiple Output (MIMO) in Wireless Local Area Networks (WLANs), IEEE has developed a Gigabit WLAN technology in IEEE 802.11 ac. Compared to its predecessor, 802.11n, $802.11 \mathrm{ac}$ is designed to provide better performance by introducing the ability of using $80 \mathrm{MHz}$ and $160 \mathrm{MHz}$ channels. However, even by using a wider RF channel, 802.11ac MIMO supports only a single transmission on the medium at one time [1].

One innovation in IEEE 802.11ac now becoming available in commercial offerings for the Wave $2802.11 \mathrm{ac}$ products is Multiple User-MIMO (MU-MIMO). With MU-MIMO, the Access Points (APs) will have the ability to send to more than one station simultaneously through beamforming techniques. Although the main goal of MU-MIMO is to provide better performance for WLANs, there are important practical considerations before deploying such technology:

- The overhead of channel estimation mechanisms required for the operation of MU-MIMO.

- Upgrading existing WLANs with MU-MIMO equipment, both APs and clients (only MU-MIMO APs were available at the time of execution of our experiments), will incur a capital cost which may or may not be justifiable according to the performance gain from such technology.

Clients systems far outnumber APs, and so one strategy for introducing MU-MIMO might be to accept the cost of replacing existing APs to support MU-MIMO, and then hope that an incremental benefit accrues as client systems are replaced through natural equipment churn. After all, this is largely what has happened for previous evolutions of 802.11. However, for $802.11 \mathrm{ac}$, we take the position that the situation is not so straight forward, and the benefits of 802.11ac may not be realised in the incremenatl fashion that has been possible for previous upgrades to the IEEE 802.11 standard. This is particularly so where legacy clients - those clients that do not support MU-MIMO - have to operate in a MU-MIMO enabled WLAN cell.

\section{A. Contribution}

We have made an empirical evaluation of the $802.11 \mathrm{ac}$ protocol with MU-MIMO using a testbed equipped with one of the latest commercial 802.11ac APs. We find that the current state of available MU-MIMO solutions (MU-MIMO APs) with legacy clients may actually lead to reduced throughput for legacy clients.

Our testbed was comprised of off-the-shelf hardware, with opensource software, and default configurations in order to demonstrate typical performance. Firstly, we conducted our study under different scenarios and assessed throughput for Transmission Control Protocol (TCP) traffic flows: TCP is the most widely used transport protocol on the Internet, used for many different applications (e.g email, WWW access and video streaming). Then we used User Datagram Protocol (UDP) traffic to identify the upper and lower boundaries of 802.11ac's performance.

Specifically, we:

- tested the performance of 802.11ac clients with and without MU-MIMO enabled at the AP.

- tested both $40 \mathrm{MHz}$ channels and $80 \mathrm{MHz}$ channels.

In keeping with the methodology of our previous work [2][8], our intentions are:

- To examine systems that are typical of normal usage, so that our results reflect real operational scenarios, rather than lab-specific, optimised configurations.

- Make it possible to apply our methodology easily to other similar scenarios, to allow comparisons.

- Allow our results to be validated / reproduced easily.

In Section II we provide background information and a rationale for our deployment scenario. In Section III we explain our methodology and describe our testbed. We present and discuss our results in Sections IV. We conclude with a short summary in Section V. 


\section{RELATED WORK AND BACKGROUND}

There have been numerous studies of both $2.4 \mathrm{GHz}$ and $5 \mathrm{GHz}$ WLAN operation. In this section, we focus on the more recent studies which are related to $5 \mathrm{GHz}$ operation and also some specific work on 802.11ac.

Our previous work [7] conducted an empirical evaluation of the performance and energy usage of different 802.11 WLANs including 802.11ac using $40 \mathrm{MHz}$ channels. We compared the performance of the 802.11ac (Wave 1) with its predecessor $802.11 \mathrm{n}$. We have examined the performance of all variants of the IEEE 802.11 family in our previous work [2]-[8]. We have also established the use of the energy metric, $E_{A}$, and the notion of the energy envelope [2], which gives the upper and lower bounds of the energy usage during the transmission of a flow. We have also investigated the possibility of application adaptation within the scope of this energy envelope [3] to trade off performance against energy usage. Also, we have found that the generic 802.11 power save mode (PSM) has little effect during system usage [4]. In [5] we address the problem of the impact on WLAN performance due to low Received Signal Strength Indication (RSSI) in WLAN cells. These studies all include measurements in both the $2.4 \mathrm{GHz}$ and $5 \mathrm{GHz}$ bands. We have also examined the energy usage of the Datagram Congestion Control protocol (DCCP) and User Datagram Protocol (UDP) at different packet sizes and data rates over $802.11 \mathrm{n}$ WLAN at $5 \mathrm{GHz}$ [6]. The results show DCCP can provide $\sim 10 \%$ to $\sim 40 \%$ greater energy efficiency than the UDP. We have compared 802.11n and 802.11ac performance, and we have also considered how different cell loads impact the performance of $5 \mathrm{GHz}$ WLAN for both 802.11n and 802.11ac [8].

Zeng et al [9] have evaluated 802.11ac performance. They observed that throughput and so energy usage was very variable, but that 802.11ac can achieve higher throughput overall when using wide RF channels $(40 \mathrm{MHz}$ and greater). The authors provided a comparison between $802.11 \mathrm{n}$ and 802.11ac protocols, but the $802.11 \mathrm{n}$ experiments used $40 \mathrm{MHz}$ channels while the $802.11 \mathrm{ac}$ experiments used $80 \mathrm{MHz}$ channels.

Diana et al [10] have empirically examined 802.11ac (Wave 1) performance in an indoor environments. They observed that 802.11ac (Wave 1) is very sensitive to channel conditions, especially when there were other 802.11 WLANs sharing the same ISM Band.

Keranidis et al [11] have considered an experimental comparison of energy efficiency for 802.11n. However, the evaluated the energy consumption for the Network Interface Card (NIC) only, while in this paper and our previous work, we consider the impact on the client system as a whole, as that will be real impact observed by users. Additionally, their study used optimisations to system configuration to improve energy efficiency, whilst we take the position that users normally adopt default configurations.

Halpern et al [12] provided an empirical study of the power consumption of 802.11n WLAN but again only considered the NIC. Their study concluded that the use of larger packets and higher date rate in transmission is more energy efficient than using a smaller packet size and lower data rates.

Li et al [13] also examined the impact of packet size on the energy consumption in heterogeneous wireless network environments, with similar observations: larger packet sizes and higher data rates give better energy efficiency.

In this study, we examine the performance of IEEE 802.11 ac (Wave 2) using TCP flows with MU-MIMO enabled at the Access Point, which has not been examined previously. Additionally, we identify the performance envelope of 802.11ac (Wave 2) using UDP flows.

\section{EXPERIMENT Design AND METRICS}

We measured performance of TCP and UDP flows transmitted over 802.11ac with and without MU-MIMO enabled at the AP. Our experiments were conducted in a modern, openplan office environment. We used opensource software, offthe-shelf hardware and default configurations for all systems, unless otherwise detailed below.

\section{A. Overview}

Our testbed (Figure 1) consisted of two clients systems. An additional host acted as a server connected via ethernet cable to a ASUS RT-AC87U ${ }^{1}$ wireless access-point (AP) which is equipped with MU-MIMO capability. The testbed was housed in a teaching lab in the University of St Andrews. The distance between the AP and client_2 was $\sim 14 \mathrm{~m}$. The other client (client_1)was placed at $\sim 5 \mathrm{~m}$ and $\sim 9 \mathrm{~m}$ from the $\mathrm{AP}$ and the client_2, respectively. iperf v2.0.2 ${ }^{2}$ was used in server mode to receive traffic flows. This measurement regime was executed with two network configurations: 802.11 ac with and without MU-MIMO using $40 \mathrm{MHz}$ and $80 \mathrm{MHz}$ channels. The TCP iperf measurements were data transfers of size $1 \mathrm{MB}$, $10 \mathrm{MB}$ and $100 \mathrm{MB}$, and were performed 30 times for each of the three transfer sizes. The experiment considered two scenarios: one where only a single client station generated TCP traffic (best case scenario); and then the first scenario was modified by adding a second station competing for the channel by transmitting UDP traffic at a mean rate of 200 Mbps. We conducted 720 measurements in total.

Additionally, we provided a detailed coverage of the performance landscape for 802.11ac with and without MU-MIMO using a $80 \mathrm{MHz}$ channel. We configured the UDP flows across a range of bit rates, with small and large packets, as shown in Table I. We chose an upper limit of $650 \mathrm{Mbps}$ data rate for a single flow, to cover a wide range of applications, and also based on our preliminary experimentation to baseline the testbed and discover its maximum throughput.

Table I shows the data rates and packet sizes used in the experiments. The 64 byte packet is the smallest size for which we have observed that iperf is able to generate server reports, and very few applications will have packets smaller than this. The 1460 byte packet is chosen as that is fits in a common MTU size used for Internet-wide communication (a known

\footnotetext{
${ }^{1}$ http://www.asus.com/uk/Networking/RTAC87U/

${ }^{2}$ http://www.erg.abdn.ac.uk/users/gerrit/dccp/apps/\#iperf
} 
TABLE I

GENERIC UDP WORKLOAD.

\begin{tabular}{|c|l|}
\hline Packet size in offered load & $64 ; 1460$ bytes \\
\hline Offered load's bit rate & $0.031 ; 0.065 ; 0.25 ; 0.5 ; 0.639 ;$ \\
& $1 ; 5 ; 10 ; 15 ; 20 ; 25 ; 30 ; 35 ;$ \\
& $40 ; 45 ; 50 ; 60 ; 70 ; 80 ; 90 ; 100 ;$ \\
& $150 ; 200 ; 250 ; 300 ; 350 ; 400 ;$ \\
& $450 ; 500 ; 550 ; 600 ; 650 \mathrm{Mbps}$ \\
\hline
\end{tabular}

Each packet size was combined with each bit-rate (64 combinations); 10 flows measured with each combination (640 flows for each); each flow had a duration of 2 minutes. The experiments runs over four different WLANs scenarios giving a total of $\sim 88$ hours of measurements.

legacy of Ethernet), and we wished to avoid the effects of IP-level fragmentation.

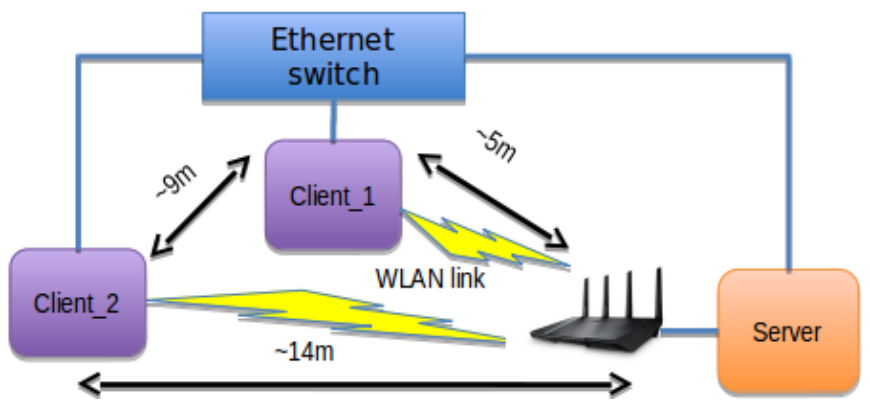

Fig. 1. Schematic of testbed showing physical connectivity. All experiments used $802.11 \mathrm{ac}$ at $5 \mathrm{GHz}$ with $40 \mathrm{MHz}$ and $80 \mathrm{MHz}$ channels. The experiments used Ethernet for a control channel and file-system access. Only test-traffic traversed the WLAN link. The antennas of the clients and access point were $\sim 14 \pm 0.5 \mathrm{~m}$ and $\sim 5 \pm 0.5 \mathrm{~m}$ apart, respectively. TCP and UDP flows generated by iperf v2.0.2 were transferred across the WLAN link.

\section{B. Equipment}

Our testbed was equipped with identical machines. The hardware specifications of the clients and the server were: a Scan home-office PC $\left(V_{10}{ }^{3}\right)$ with an Intel $\AA$ Core i5 4440 3.1GHz Quad Core CPU, 4GB RAM, 1TB HD. All clients used the same wireless LAN NIC hardware ${ }^{4}$ based on the QCA9880 Version 2 Atheros ${ }^{5}$ chipset, with $3 \times 3$ MIMO technology. The server is connected with the MU-MIMOcapable AP, and ASUS RT-AC87U ${ }^{6}$, with $4 \times 4$ MU-MIMO technology.

Ubuntu 14.04 was used on each host, a minimal server distribution, with the Linux kernel version 3.16.0-wl-ath+, which used the latest ath10k driver ${ }^{7}$. All nodes in the testbed were connected via a local Ethernet network, which was also used for controlling the experiment: only test traffic traversed the WLAN link. The Linux utility iwconfig ${ }^{8}$ was used to record the link quality and the signal level of the RF channel.

\footnotetext{
${ }^{3}$ http://3xs.scan.co.uk/configurator/ready-to-ship-budget-value-amd-homeoffice-pc-v10a

${ }^{4}$ http://www.compex.com.sg/Datasheets/WLE900VX_Dsv1.0.1-140711I.pdf

${ }^{5}$ http://www.atheros.com/

${ }^{6} \mathrm{http}: / /$ www.asus.com/uk/Networking/RTAC87U/

${ }^{7}$ https://github.com/kvalo/ath10k

${ }^{8}$ http://www.linuxcommand.org/man_pages/iwconfig8.html
}

\section{RESUlTS AND Discussion}

In this section we provide the analyses and discussion of the results obtained from our experiments. As variability in performance can be high (as also observed in [10]), in each TCP measurement's graph we show:

- All 30 measurements summarised as a standard boxplot (minimum whisker, 25th-percentile, median, 75thpercentile, maximum whisker).

- Offset to the right of the boxplot, for each set of 30 measurements, we plot a point for the mean value, with a whisker showing the 95th-percentile and 99th-percentile.

The experiments focused on two scenarios in order to evaluate the MU-MIMO capability in an office space. Section IV-A discusses the result of a single active TCP client communicating via the AP, with no other stations competing for the channel. The basic idea behind conducting experiments using only a single active station is to evaluate the practical overhead of introducing MU-MIMO.

Section IV-B discusses the results obtained from the testbed when the initial scenario was modified, and a second client sent a stream of UDP packets at a mean rate of of 200Mbps, competing for the available channel. This scenario was designed to examine the MU-MIMO capability of potentially supporting more than one client simultaneously, but where the clients themselves are are not MU-MIMO capable.

In addition to the TCP performance measurements, sectionIV-C provides a performance envelope for $802.11 \mathrm{ac}$ using $80 \mathrm{MHz}$ channels with and without using MU-MIMO, which gives the upper and lower bounds of the gains that would be possible. Sections IV-D and IV-E, discuss MUMIMO overhead and legacy clients. Section IV-F identifies some limitations of the study.

\section{A. Single active station scenario}

Figures 2 and 4 show the throughput of the TCP flows for a single active station connected to the $\mathrm{AP}$, for $40 \mathrm{MHz}$ and 80 $\mathrm{MHz}$ channels, respectively. From the figures, we can observe that 802.11 ac can peak at $\sim 700 \mathrm{Mbps}$ in some cases (Figure $4 \mathrm{~b}$ for example) and up to $450 \mathrm{Mbps}$ on average for the $80 \mathrm{MHz}$ channel without MU-MIMO in operation. This is considered the best case scenario where there is no competition for the shared media, and the AP uses the widest available available RF channel. For the $100 \mathrm{MB}$ transfer, Figure $4 \mathrm{c}$, the maximum achieved throughput was $\sim 500 \mathrm{Mbps}$.

Key observation: Although 802.11ac is designed to be a Gigabit WLAN solutions, in practice, mean rates of transfer achieved are at about half of this rate (as has been observed for other 802.11 variants in the past).

Considering results when MU-MIMO was in operation compared to when it is turned off, Figures 2 and 4, it is clear that the WLAN cell had better mean throughput when MU-MIMO was not in use. As the main goal of MU-MIMO is to provide two or more active station with concurrent communication, using this technology for a single station scenario will introduce some overhead and result in degraded 
performance. Section IV-D will provide more discussion about the MU-MIMO overhead.

\section{B. Two active stations scenario}

This scenario consisted of two active clients connected to the AP. One client, at $\sim 5 \mathrm{~m}$ from the AP, generated UDP background traffic of $200 \mathrm{Mbps}$ to emulate the activity of other WLAN clients in a busy cell. Figures 3 and 5 illustrate the results of the TCP flow throughput in the presence of this background traffic. The results show both cases of the WLAN cell - with and without MU-MIMO - using $40 \mathrm{MHz}$ and $80 \mathrm{MHz}$ channels. The scenario was designed to test the capability of MU-MIMO 802.11ac when providing service for two legacy clients with two stations at the same time. As shown in the figures, for both $40 \mathrm{MHz}$ and $80 \mathrm{MHz}$ channels, there is no evidence of performance improvement when MU-MIMO was enabled. Surprisingly, the performance of the WLAN cell was slightly better on average in many cases when MU-MIMO was not in use. This is because the clients have no MU-MIMO functionality (wireless cards with MUMIMO functionality were unavailable at the time of writing this paper). So relying on the MU-MIMO APs to improve the WLAN cell's performance without upgrading the clients is not an effective choice, and it may reduce the WLAN cell's performance rather than improving it.

Key observation: Deploying MU-MIMO APs without MUMIMO clients cannot improve the performance of the WLAN and may be deterimental to legacy (non-MU-MIMO) clients.

\section{802.11ac Maximum Performance}

In all the Figures in this subsection, we plot the mean of the runs, and plot standard error bars (95\% confidence), but in the majority of the experiments, only very small error bars were calculated, so they may not always be easily visible even though they have been plotted.

Figures 6,7 and 8 show the performance envelope of 802.11 ac protocol with and without using MU-MIMO. This envelope indicates the upper and lower bounds of 802.11ac performance. We examined the performance of the 802.11ac protocol using $80 \mathrm{MHz}$ channel as our aim was to identify the maximum performance that can be achieved by $802.11 \mathrm{ac}$. Figure 6 shows throughput of 802.11ac with a single active station. The maximum throughput that 802.11ac achieved for flows of small packets ( 64 byte packet size) was $\sim 35$ Mbps. For the flows of large packets (1460 byte packet size), 802.11 ac achieved up to $\sim 580$ Mbps.

Figures 7 and 8 show the performance of 802.11 ac for two active stations. For the station at $\sim 14 \mathrm{~m}$ distnace from the AP client (see Figure 1), figure 7 shows that the maximum achieved throughput was $\sim 300 \mathrm{Mbps}$ for flows of large packet with MU-MIMO on and MU-MIMO off. Also, the figure shows that for flows of small packet flows the mean througput is $\sim 35 \mathrm{Mbps}$ for MU-MIMO off, but for MU-MIMO on, the mean throughput is $\sim 20 \mathrm{Mbit} / \mathrm{s}$ with more variability. For the station at $\sim 5 \mathrm{~m}$ from the AP client, figure 8 shows no difference in the performance whether the MU-MIMO is on or off.

\section{MU-MIMO Ooverhead}

Beamforming is the main technology behind MU-MIMO to allows two or more active stations to receive / send data at the same time. However, for effective beamforming, the beamformer (AP) needs a very accurate estimation of the channel to each of the beamformees (the clients) by introducing an explicit channel feedback mechanism. In order to achieve a high throughput, a MU-MIMO AP needs to operate at 64QAM and 256-QAM modulation schemes, which require a feedback signal for channel estimation at frequent intervals (in the order of milliseconds). This will introduce overhead and reduce the performance of the whole WLAN cell, especially when legacy (non-MU-MIMO) are using the WLAN.

\section{E. MU-MIMO and legacy clients}

As the current state of MU-MIMO solutions operates in the downstream direction, from the access point to the client, the results show that there is no improvement with the legacy clients. The only scenario that may achieve overall WLAN cell throughput improvement is when there are more MU-MIMO active client stations using the WLAN cell. In this scenario, taking advantage of boht MU-MIMO capable AP and clients, the WLAN could have capacity to serve other legacy clients, whilst still serving the MU-MIMO clients via beamforming.

So, perhaps we simply need to wait for all client systems to become MU-MIMO capable also? For the short- to mediumterm, at least, this is not going to be the case. This is because it is only now that $802.11 \mathrm{ac} 5 \mathrm{GHz}$ is being seen in new products as a regular product feature (indeed, some client systems, in order to keep costs low, still only support $802.11 \mathrm{n}$ at $2.4 \mathrm{GHz}$ ). So, it may be some time before costs of 802.11ac Wave 1, and then Wave 2, equipment are reduced so that more devices can use MU-MIMO.

However, unless there are innovations in antenna technology and coding mechanisms, it may be that MU-MIMO may not appear in smaller equipment. This is because smaller devices, such as smartphones, are constrained on physical size, battery power and costs. So, geometry (restricted spatial diversity), lack of power and costs of components may restrict the deployment of MU-MIMO, meaning that legacy (nonMU-MIMO) devices will be present in MU-MIMO enabled networks for a long time to come.

\section{F. Limitations of our study}

The clients used in the testbed were non MU-MIMO clients, as MU-MIMO clients are not yet available. In the presence of a MU-MIMO client, performance may be better for a legacy client, as the MU-MIMO AP could serve the MU-MIMO client using beamforming, whilst also serving the legacy client. The testbed used two stations, and the scenarios used consisted of either a single active station or two active stations, the second emulating background cell traffic. Multiple stations may create a different background traffic profile, but it is difficult to find a 


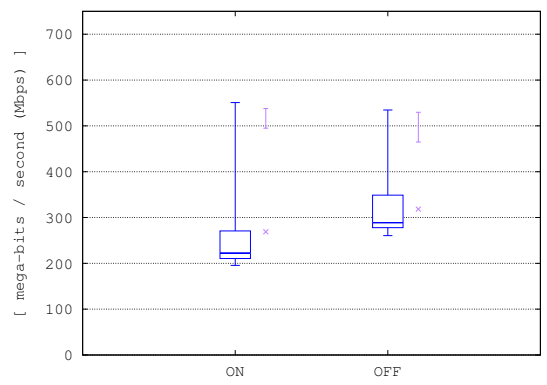

(a) $1 \mathrm{MB}$ Transfer

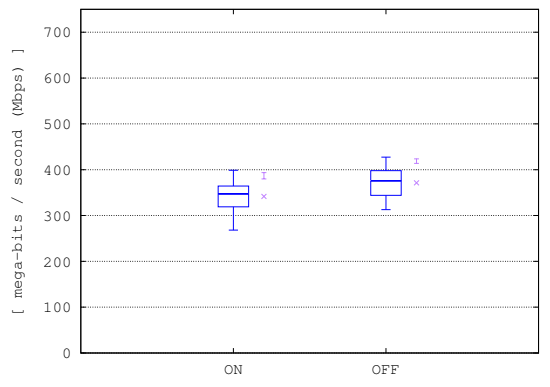

(b) $10 \mathrm{MB}$ Transfer

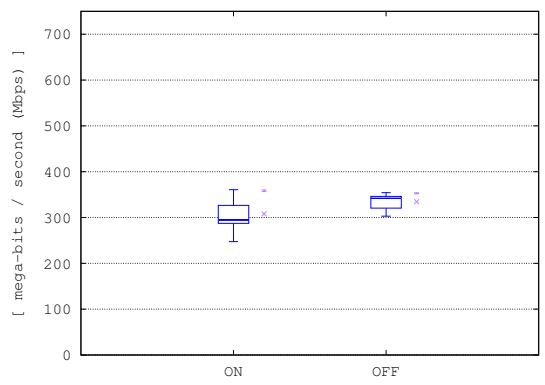

(c) $100 \mathrm{MB}$ Transfer

Fig. 2. Single station scenarios: Throughput for TCP flows for $802.11 \mathrm{ac}$ using $40 \mathrm{MHz}$ channel width.

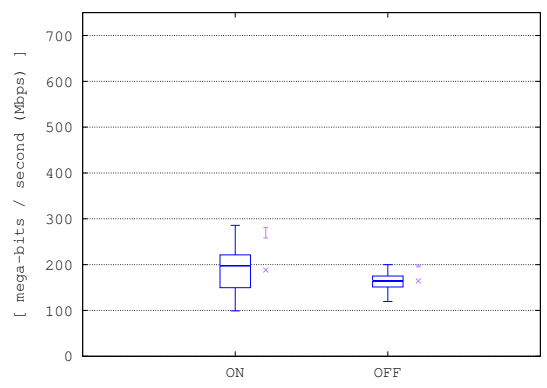

(a) 1 MB Transfer

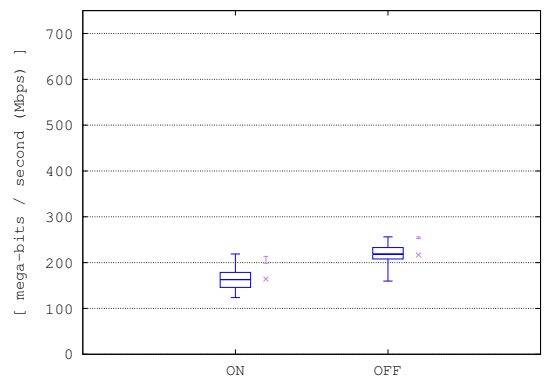

(b) $10 \mathrm{MB}$ Transfer

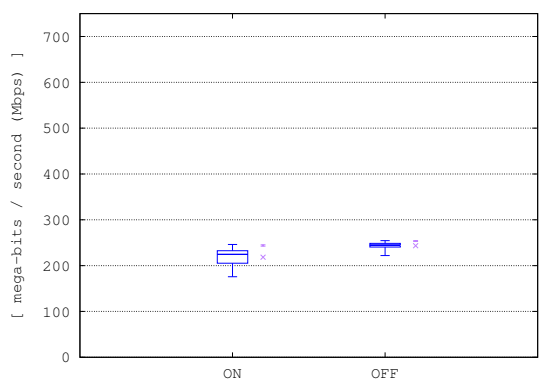

(c) $100 \mathrm{MB}$ Transfer

Fig. 3. Two stations scenarios: Throughput for TCP flows for $802.11 \mathrm{ac}$ using $40 \mathrm{MHz}$ channel width.

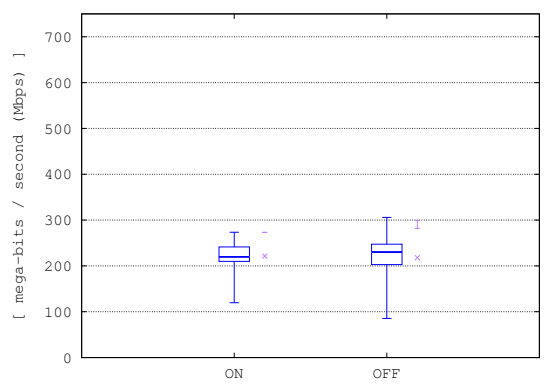

(a) 1 MB Transfer

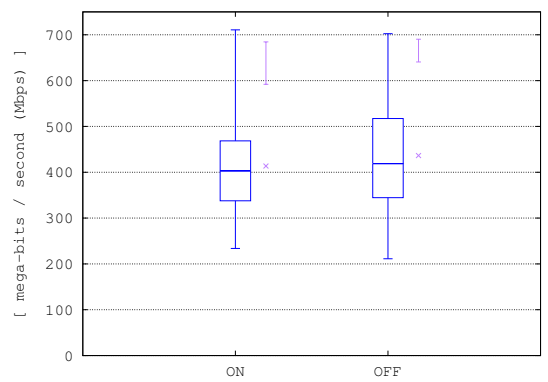

(b) $10 \mathrm{MB}$ Transfer

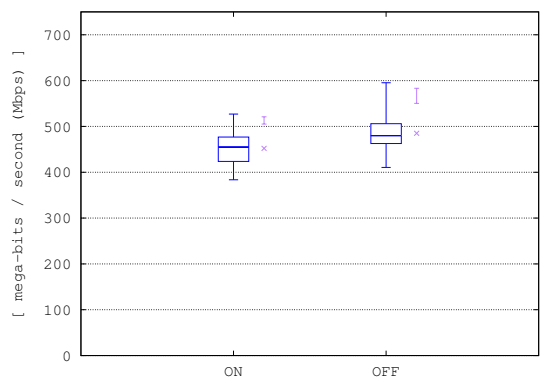

(c) $100 \mathrm{MB}$ Transfer

Fig. 4. Single station scenarios: Throughput for TCP flows for 802.11 ac using $80 \mathrm{MHz}$ channel width.

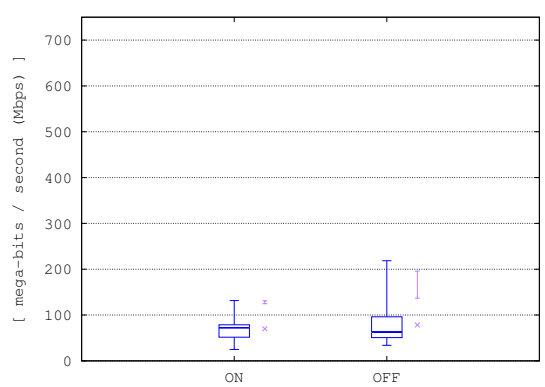

(a) 1 MB Transfer

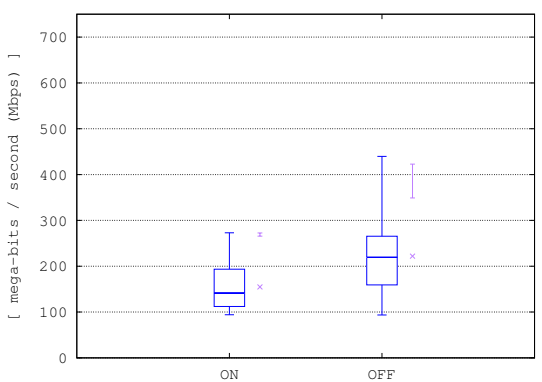

(b) $10 \mathrm{MB}$ Transfer

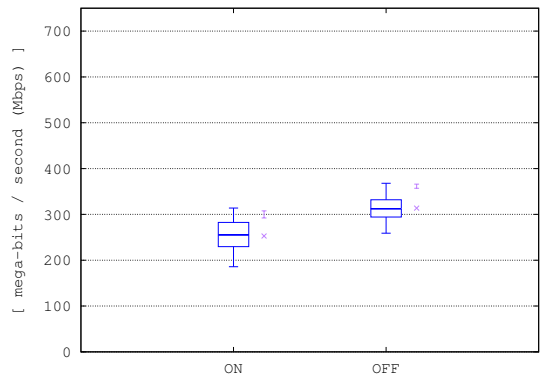

(c) $100 \mathrm{MB}$ Transfer

Fig. 5. Two stations scenarios: Throughput for TCP flows for $802.11 \mathrm{ac}$ using $80 \mathrm{MHz}$ channel width. 


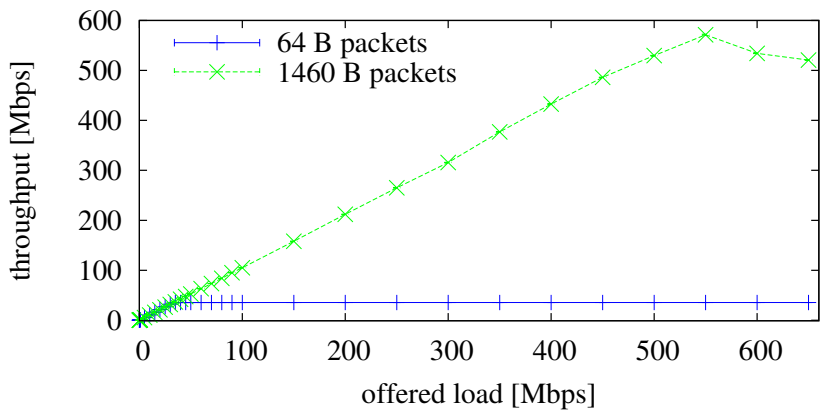

(a) MU-MIMO ON

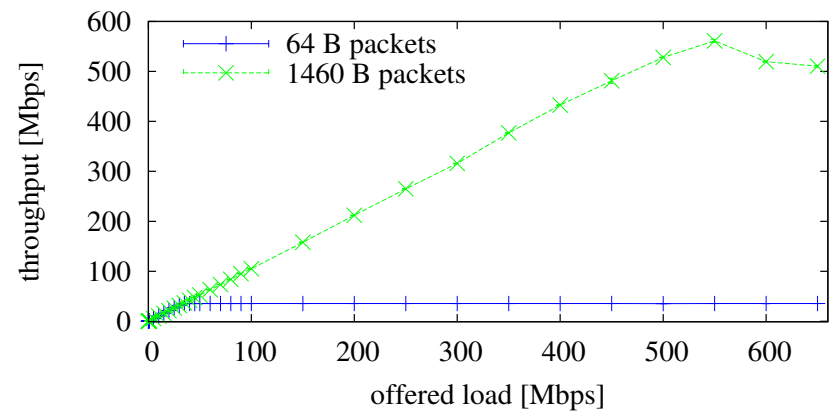

(b) MU-MIMO OFF

Fig. 6. Single station scenarios: Throughput for UDP flows for $802.11 \mathrm{ac}$ using $80 \mathrm{MHz}$ channel width.

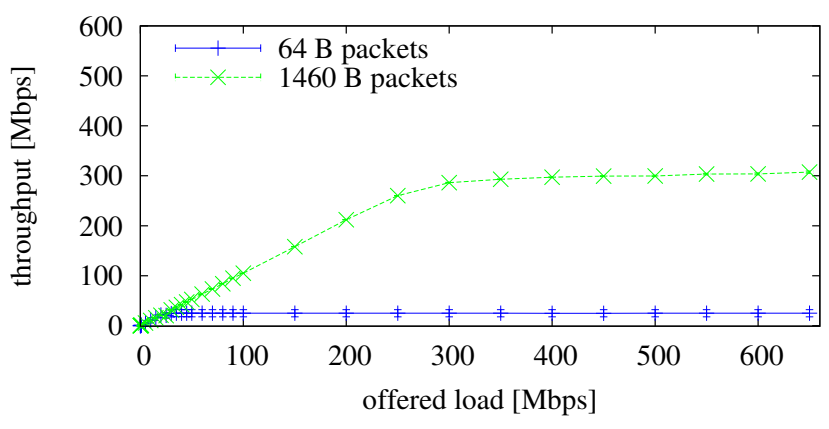

(a) MU-MIMO ON

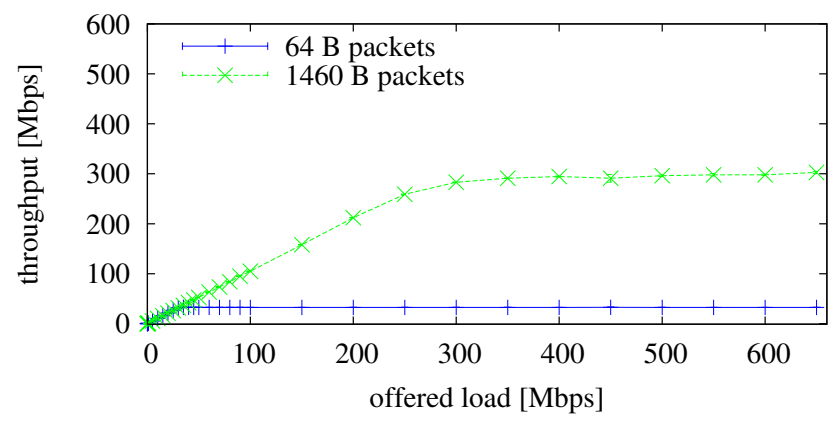

(b) MU-MIMO OFF

Fig. 7. Two stations scenarios: [far away from the AP client] Throughput for UDP flows for $802.11 \mathrm{ac}$ using $80 \mathrm{MHz}$ channel width.

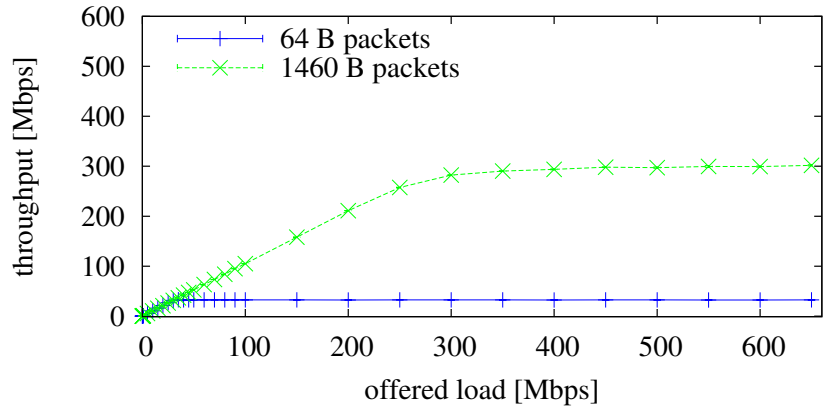

(a) MU-MIMO ON

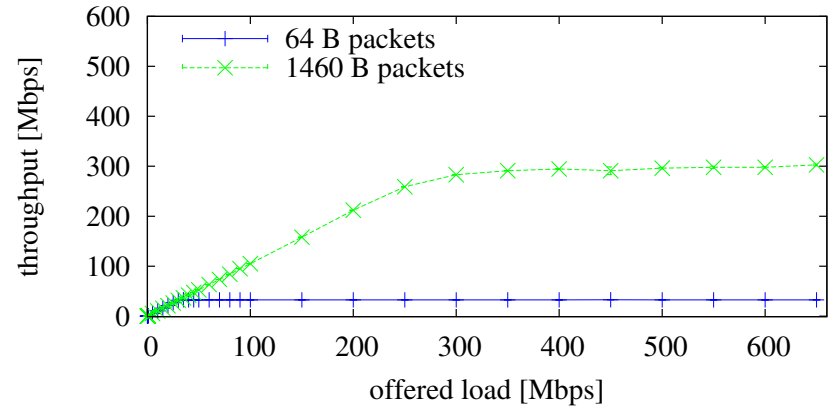

(b) MU-MIMO OFF

Fig. 8. Two stations scenarios: [closest to AP client] Throughput for UDP flows for $802.11 \mathrm{ac}$ using $80 \mathrm{MHz}$ channel width.

profile of an 'average' representative WLAN cell (all situations are different). Our testbed measured transmission between the access point and a client directly connected via the WLAN link. We do not consider an Internet-wide end-to-end path. The applicability of our measurements is therefore on a local access scenario, where the client systems serve users with access to local systems. However, that relates well to many scenarios, including a home system, and office system, or a campus scenario. In the office and campus, many resources are local and there may be institutional caching, which again mataches well with our testbed set-up.

If an Internet-wide end-to-end path was considered, performance bottlenecks and/or packet loss along paths with higher round trip times than our testbed would yield different results for TCP. Of course, our intention was to examine the 802.11ac performance and not necessarily the performnace of TCP or of the network as a whole.

\section{CONClusion}

We have conducted experiments to measure the performance of IEEE 802.11ac WLAN with MU-MIMO technology at 
the access point, but with non-MU-MIMO/legacy clients. Our experiments considered using $40 \mathrm{Mhz}$ and $80 \mathrm{MHz}$ channels with two scenarios: a single station and a single station with background traffic emulating a busy cell.

We find that with our non-MU-MIMO/legacy 802.11ac clients, using MU-MIMO at the AP has no clear advantage in performance compared to 802.11ac without MU-MIMO, and indeed, MU-MIMO may result in poorer performance for the non-MU-MIMO/legacy clients. So, upgrading existing IEEE 802.11 n $5 \mathrm{GHz}$ infrastructure deployments to IEEE $802.11 \mathrm{ac}$ MU-MIMO are likley to yield limited benfits overall, until client systems are also upgraded to take advantage of the the MU-MIMO capability that is possible.

\section{ACKNOWLEDGEMENTS}

This work has been supported by the IU-ATC project (http://www.iu-atc.com/), funded by grant EP/J016756/1, awarded by the Engineering and Physical Sciences Research Council (EPSRC), UK.

\section{REFERENCES}

[1] M. Park, "IEEE 802.11ac: Dynamic Bandwidth Channel Access," in Communications (ICC), 2011 IEEE International Conference on, June 2011, pp. 1-5.

[2] M. Tauber, S. N. Bhatti, and Y. Yu, "Application Level Energy and Performance Measurements in a Wireless LAN," in GreenCom2011 IEEE/ACM Intl. Conf. Green Computing and Comms., Aug 2011.

[3] M. Tauber and S. N. Bhatti and Y. Yu, "Towards Energy-Awareness in Managing Wireless LAN Applications," in NOMS 2012 - IEEE/IFIP Network Operations and Management Symp., Apr 2012.
[4] M. Tauber and S. N. Bhatti, "The Effect of the 802.11 Power Save Mechanism (PSM) on Energy Efficiency and Performance During System Activity," in GreenCom 2012 - IEEE Intl. Conf. Green Computing and Communications, Nov 2012, pp. 573-580.

[5] M. Tauber and S. Bhatti, "Low RSSI in WLANs: Impact on applicationlevel performance," in ICNC2013 - 2013 Intl. Conf. on Computing, Networking and Communications, 2013, pp. 123-129.

[6] M. Abu-Tair and S. N. Bhatti, "Energy Usage of UDP and DCCP over 802.11n," in IEEE AINA 2014 - 28th Intl. Conf. Advanced Information Networking and Applications, 2014, pp. 313-320.

[7] _ - "Upgrading 802.11 deployments: A critical examination of performance," in AINA2015 - 29th IEEE Intl. Conf.on Advanced Information Networking and Applications, March 2015, pp. 844-851.

[8] — "Impact of cell load on 5GHz IEEE 802.11 WLAN," in PAEWN2017 - 12th Intl. Wrkshp. on the Performance Analysis and Enhancement of Wireless Networks, Mar 2017.

[9] Y. Zeng, P. H. Pathak, and P. Mohapatra, "A First Look at 802.11ac in Action: Energy Efficiency and Interference Characterization," in Networking2014 - IFIP Intl. Conf. on Networking, Jun 2014.

[10] M.-D. Dianu, J. Riihijarvi, and M. Petrova, "Measurement-based study of the performance of ieee 802.11ac in an indoor environment," in Communications (ICC), 2014 IEEE International Conference on, June 2014, pp. 5771-5776.

[11] S. Keranidis, G. Kazdaridis, N. Makris, T. Korakis, I. Koutsopoulos, and L. Tassiulas, "Experimental Evaluation and Comparative Study on Energy Efficiency of the Evolving IEEE 802.11 Standards," in Proc. e-energy2014 - 5th ACM Intl. Conf. on Future Energy Systems, ser. e-Energy '14. New York, NY, USA: ACM, 2014, pp. 109-119. [Online]. Available: http://doi.acm.org/10.1145/2602044.2602069

[12] D. Halperin, B. Greensteiny, A. Shethy, and D. Wetherall, "Demystifying $802.11 \mathrm{n}$ power consumption," in 2010 Wkshp. on Power Aware Computing and Systems, ser. HotPower'10. Berkeley, CA, USA: USENIX Association, 2010. [Online]. Available: http://portal.acm.org/citation.cfm?id=1924920.1924928

[13] Y. Li, X. Qi, M. Keally, Z. Ren, G. Zhou, D. Xiao, and S. Deng, "Communication Energy Modeling and Optimization through Joint Packet Size Analysis of BSN and WiFi Networks," IEEE Trans. Parallel and Distributed Systems, vol. 24, no. 9, pp. 1741-1751, 2013. 